\title{
Hunting for the non-Hermitian exceptional points with fidelity susceptibility
}

\author{
Yu-Chin Tzeng $\odot,{ }^{1,2,3,{ }^{*}}$ Chia-Yi Ju $\odot,{ }^{3}$ Guang-Yin Chen $\odot,{ }^{3, \dagger}$ and Wen-Min Huang ${ }^{3, *}$ \\ ${ }^{1}$ Department of Physics, National Tsing Hua University, Hsinchu 30013, Taiwan \\ ${ }^{2}$ Department of Applied Physics, Tunghai University, Taichung 40704, Taiwan \\ ${ }^{3}$ Department of Physics, National Chung Hsing University, Taichung 40227, Taiwan
}

(Received 13 September 2020; revised 1 November 2020; accepted 22 December 2020; published 7 January 2021)

\begin{abstract}
The fidelity susceptibility has been used to detect quantum phase transitions in the Hermitian quantum many-body systems over a decade, where the fidelity susceptibility density approaches $+\infty$ in the thermodynamic limits. Here the fidelity susceptibility $\chi$ is generalized to non-Hermitian quantum systems by taking the geometric structure of the Hilbert space into consideration. Instead of solving the metric equation of motion from scratch, we chose a gauge where the fidelities are composed of biorthogonal eigenstates and can be worked out algebraically or numerically when not on the exceptional point (EP). Due to the properties of the Hilbert space geometry at the EP, we found that the EP can be found when $\chi$ approaches $-\infty$. As examples, we investigate the simplest $\mathcal{P} \mathcal{T}$ symmetric $2 \times 2$ Hamiltonian with a single tuning parameter and the non-Hermitian Su-Schriffer-Heeger model.
\end{abstract}

DOI: 10.1103/PhysRevResearch.3.013015

\section{INTRODUCTION}

The overlap of two states, or fidelity $\mathcal{F}_{\mathrm{h}}(|\psi\rangle,|\varphi\rangle)=$ $\langle\psi \mid \varphi\rangle\langle\varphi \mid \psi\rangle$ to be more specific, is used in the quantum information sciences as an estimation of the similarity of two quantum states. In quantum many-body system, the eigenstate fidelity and the fidelity susceptibility have been used to detect quantum phase transitions [1]. Away from the critical point, two ground states with nearby parameters in the same phase are expected to have a high fidelity or a finite value of fidelity susceptibility. On the other hand, when the system is close to the quantum critical point, a quick drop in the fidelity or divergence in fidelity susceptibility density is very likely to occur. Although the definition of the fidelity is far from unique, e.g., the square root of $\mathcal{F}_{\mathrm{h}}$ is also widely accepted, as a quantity that defines the closeness of two states, the fidelity satisfies the Jozsa's axioms [2]:

(i) $0 \leqslant \mathcal{F}_{\mathrm{h}}(|\psi\rangle,|\varphi\rangle) \leqslant 1$;

(ii) $\mathcal{F}_{\mathrm{h}}(|\psi\rangle,|\varphi\rangle)=\mathcal{F}_{\mathrm{h}}(|\varphi\rangle,|\psi\rangle)$;

(iii) $\mathcal{F}_{\mathrm{h}}(U|\psi\rangle, U|\varphi\rangle)=\mathcal{F}_{\mathrm{h}}(|\psi\rangle,|\varphi\rangle)$, where $U$ is an arbitrary unitary operator; and

(iv) $\mathcal{F}_{\mathrm{h}}\left(\left|\psi_{1}\right\rangle \otimes\left|\psi_{2}\right\rangle, \quad\left|\varphi_{1}\right\rangle \otimes\left|\varphi_{2}\right\rangle\right)=\mathcal{F}_{\mathrm{h}}\left(\left|\psi_{1}\right\rangle, \quad\left|\varphi_{1}\right\rangle\right) \mathcal{F}_{\mathrm{h}}$ $\left(\left|\psi_{2}\right\rangle,\left|\varphi_{2}\right\rangle\right)$.

Since the discovery of that a non-Hermitian Hamiltonian with parity-inversion $(\mathcal{P})$ plus time-reversal $(\mathcal{T})$ symmetry has

\footnotetext{
*yctzeng@mx.nthu.edu.tw

†gychen@phys.nchu.edu.tw

${ }^{\ddagger}$ wenmin@phys.nchu.edu.tw
}

Published by the American Physical Society under the terms of the Creative Commons Attribution 4.0 International license. Further distribution of this work must maintain attribution to the author(s) and the published article's title, journal citation, and DOI. real eigenvalues [3], it has been attracting both theoretical and experimental investigations on the non-Hermitian systems in many fields, ranging from classical optics, acoustics, optomechanics, mechanics, electronics, metamaterials, plasmonics, condensed matter physics, and photonic crystals to innovative devices [4-18]. However, the fidelity $\mathcal{F}_{\mathrm{h}}$ losses its meaning in the non-Hermitian quantum systems because the inner product in conventional quantum mechanics leads to all kinds of weird behaviors, e.g., faster-than-light communication [19], and entanglement increasing under local operations and classical communications (LOCC) [20]. To generalize the fidelity for non-Hermitian systems, it is necessary to use a proper inner product. Utilizing the geometric meaning of Schrödinger's equation, one finds a proper inner product that is free from the above mentioned weird behavior. Rather than being a strictly Euclidean-like Hilbert space, the Hilbert space of the quantum states can have its own "geometry" or metric [21-23].

Given a non-Hermitian Hamiltonian $H \neq H^{\dagger}$, the connection-compatible metric operator $G$ of the system has to be positive-definite, $G=G^{\dagger}$, and satisfy the equation of motion:

$$
\frac{\partial}{\partial t} G=i\left(G H-H^{\dagger} G\right)
$$

The corresponding dual vector of $|\psi\rangle$ becomes $\langle\langle\psi|=\langle\psi| G$. For notation consistency, define $|\varphi\rangle\rangle=|\varphi\rangle$ and the inner product becomes $\langle\langle\psi \mid \varphi\rangle\rangle=\langle\psi|G| \varphi\rangle$. The detailed derivation can be found in Ref. [23]. It is known that if the Hamiltonian is not at the exceptional point, then the metric can always be chosen as

$$
G=\sum_{i}\left|L_{i}\right\rangle\left\langle L_{i}\right| .
$$


Where $\left|L_{i}\right\rangle$ is the $i$ th left eigenvector of the Hamiltonian, i.e., $\left\langle L_{i}\right| H=\left\langle L_{i}\right| E_{i}$. This gauge choice [23] not only reproduces the biorthogonal quantum mechanics [24], it also works best with Hamiltonian eigenstates. Note that the $j$ th right eigenvector $\left|R_{j}\right\rangle$ satisfies $H\left|R_{j}\right\rangle=E_{j}\left|R_{j}\right\rangle$ and the biorthonormal relation $\left\langle L_{i} \mid R_{j}\right\rangle=\delta_{i j}$.

The exceptional point (EP) [25-28] of the non-Hermitian systems is a special point in the parameter space where both eigenvalues and eigenstates merge into only one value and state. In general, the eigenstates of a non-Hermitian Hamiltonian are not orthogonal with each other using the conventional inner product. As the parameter approaches the EP, two or more eigenstates close to each other and eventually coalesce into one. Thus, the Hamiltonian at the EP has less eigenvectors and cannot span the entire Hilbert space. The EPs are also associated with the real-to-complex spectral transition for parity-time $(\mathcal{P} \mathcal{T})$ symmetric Hamiltonians [26]. The EPs provide genuine singularities, which can manifest prominently in optical [29], plasmonic [30], and microwave [31] response properties and hybrid dynamical systems [32-34], cold atoms [35], correlated systems [36,37], sensing enhancement, and scattering problems [38].

Since finding the coalescence of eigenvectors by numerical full diagonalization can be tedious work, theoretical development in detecting the EP becomes interesting. Here the fidelity susceptibility is used to detect these interesting points. Although there are few non-Hermitian generalizations of fidelity suggested in the literature very recently $[39,40]$, we propose a more natural generalization by taking the aforementioned Hilbert space geometry into account.

\section{GENERALIZED FIDELITY SUSCEPTIBILITY}

In this paper the fidelity is generalized for comparing both the eigenstates and the metrics in the non-Hermitian Hamiltonian with one tunable parameter, e.g., $H(\lambda)=H_{0}+\lambda H^{\prime}$, with one of the left and right eigenvector pair, $|L(\lambda)\rangle$ and $|R(\lambda)\rangle$, with $\langle L(\lambda) \mid R(\lambda)\rangle=1$. We define the generalized (metricized) fidelity

$$
\mathcal{F}:=\langle\langle R(\lambda) \mid R(\lambda+\epsilon)\rangle\rangle\langle\langle R(\lambda+\epsilon) \mid R(\lambda)\rangle\rangle,
$$

where $\quad\langle\langle R(\lambda)|=\langle R(\lambda)| G(\lambda) \quad$ and $\quad\langle\langle R(\lambda+\epsilon)|=$ $\langle R(\lambda+\epsilon)| G(\lambda+\epsilon)$. Although this definition does not necessarily satisfy the first Josza's axiom due to the geometry deformation in the Hilbert spaces, it is easy to see that when $H=H^{\dagger}, G=\mathbb{1}$ is Hermitian, positive-definite, and a solution to Eq. (1). Therefore, $\mathcal{F}$ reduces back to $\mathcal{F}_{\mathrm{h}}$ in Hermitian systems. While the generalized fidelity $\mathcal{F}$ compares states not only in different geometry but also in the time evolution, $\mathcal{F}$ is constant in time. Using the Schrödinger's equation together with Eq. (1), the time derivative on the generalized fidelity vanishes trivially,

$$
\frac{\partial}{\partial t} \mathcal{F}=0
$$

Therefore, this generalized fidelity can indeed be used to quantify the closeness between the states in different geometries of Hilbert space. Choosing the metric found in Eq. (2) is equivalent to compute the left and right eigenstates of the
Hamiltonian as long as both $\lambda$ and $\lambda+\epsilon$ are not the EP [41],

$$
\mathcal{F}=\langle L(\lambda) \mid R(\lambda+\epsilon)\rangle\langle L(\lambda+\epsilon) \mid R(\lambda)\rangle .
$$

The generalized fidelity can be expanded as $\mathcal{F}=1-$ $\chi \epsilon^{2}+O\left(\epsilon^{3}\right)$ if the parameter $\lambda$ is not an EP and $\epsilon$ is sufficiently small. The generalized fidelity susceptibility $\chi$ can be approximated as

$$
\chi \approx \frac{1-\mathcal{F}}{\epsilon^{2}} .
$$

The fact that $\mathcal{F}$ reduces back to $\mathcal{F}_{\mathrm{h}}$ in Hermitian cases means that the generalized fidelity susceptibility $\chi$ can also be used to detect the phase transition, where the fidelity susceptibility density tends to $+\infty$ at the critical point [42]. Moreover, since $\mathcal{F}(\lambda)$ is affected by the difference between the metrics $G(\lambda)$ and $G(\lambda+\epsilon)$, the susceptibility $\chi(\lambda)$ is expected to diverge when the Hilbert space is changing its phase. Furthermore, since the sole reason that $\mathcal{F}(\lambda)$ can sit outside of region $[0,1]$ is the metric difference in the Hilbert space, the "geometric phase change" is very likely to occur as $|\mathcal{F}(\lambda)|>1$. Since $G(\lambda)$ changes smoothly except at the EPs [23], together with the aforementioned properties, the limit of $\operatorname{Re} \chi(\lambda)$ should tend to negative infinity when $\lambda$ approaches an $\operatorname{EP}(\tilde{\lambda})$, i.e.,

$$
\lim _{\lambda \rightarrow \tilde{\lambda}} \operatorname{Re} \chi(\lambda)=-\infty
$$

\section{EXAMPLES}

\section{A. A toy model}

Here we provide two examples demonstrating the previous statement or Eq. (7). The simplest single variable adjustable Hamiltonian with an EP is a $\mathcal{P} \mathcal{T}$-symmetric Hamiltonian [43],

$$
H(r)=\left[\begin{array}{cc}
i r & 1 \\
1 & -i r
\end{array}\right],
$$

where $r \in \mathbb{R}$ is the adjustable parameter. The eigenstates coalesce at $r= \pm 1$; namely, $r= \pm 1$ is an EP of the Hamiltonian. Moreover, the $\mathcal{P} \mathcal{T}$ symmetry is preserving as $|r|<1$; however, $\mathcal{P} \mathcal{T}$ symmetry is breaking as $|r|>1$.

In the $\mathcal{P} \mathcal{T}$ preserving region $(|r|<1)$, the time-evolving right eigenstates are

$$
\begin{aligned}
\left.\left|\psi_{1}(t)\right\rangle\right\rangle= & \frac{\exp \left(-i \sqrt{1-r^{2}} t\right)}{\sqrt{2 \cos \alpha}}\left[\begin{array}{c}
e^{i \alpha / 2} \\
e^{-i \alpha / 2}
\end{array}\right], \\
\left.\left|\psi_{2}(t)\right\rangle\right\rangle= & \frac{i \exp \left(i \sqrt{1-r^{2}} t\right)}{\sqrt{2 \cos \alpha}}\left[\begin{array}{c}
e^{-i \alpha / 2} \\
-e^{i \alpha / 2}
\end{array}\right],
\end{aligned}
$$

where $\cos \alpha=\sqrt{1-r^{2}}$. By using Eq. (2) the corresponding metric is

$$
G=\frac{1}{\cos \alpha}\left[\begin{array}{cc}
1 & -i \sin \alpha \\
i \sin \alpha & 1
\end{array}\right] .
$$

In the $\mathcal{P} \mathcal{T}$ breaking region $(|r|>1)$, the time-evolving right eigenstates are

$$
\begin{aligned}
\left.\left|\psi_{1}(t)\right\rangle\right\rangle & =\frac{\exp (t \Lambda)}{\sqrt{2 r \Lambda-2 \Lambda^{2}}}\left[\begin{array}{c}
1 \\
-i(r-\Lambda)
\end{array}\right], \\
\left.\left|\psi_{2}(t)\right\rangle\right\rangle & =\frac{\exp (-t \Lambda)}{\sqrt{2 r \Lambda-2 \Lambda^{2}}}\left[\begin{array}{c}
i(r-\Lambda) \\
1
\end{array}\right],
\end{aligned}
$$


where $\Lambda=\sqrt{r^{2}-1}$. Then the corresponding metric becomes

$$
G=\frac{1}{2 \Lambda}\left[\begin{array}{ll}
g_{11} & g_{12} \\
g_{21} & g_{22}
\end{array}\right],
$$

where $\quad g_{11}=(\Lambda+r) e^{-2 t \Lambda}-(\Lambda-r) e^{2 t \Lambda}, \quad g_{22}=(\Lambda+$ $r) e^{2 t \Lambda}-(\Lambda-r) e^{-2 t \Lambda}, \quad g_{12}=-i\left(e^{2 t \Lambda}+e^{-2 t \Lambda}\right), \quad g_{21}=$ $i\left(e^{2 t \Lambda}+e^{-2 t \Lambda}\right)$.

In both regions, the expansion of the fidelity $\mathcal{F}(r)=$ $\left\langle\left\langle\psi_{1}(r) \mid \psi_{1}(r+\epsilon)\right\rangle\right\rangle\left\langle\left\langle\psi_{1}(r+\epsilon) \mid \psi_{1}(r)\right\rangle\right\rangle$ to the $\epsilon^{2}$ order is

$$
\mathcal{F}(r)=1+\frac{1}{4\left(1-r^{2}\right)^{2}} \epsilon^{2}+O\left(\epsilon^{3}\right)
$$

The fidelity is time independent, and the fidelity susceptibility is

$$
\chi(r)=\frac{-1}{4\left(1-r^{2}\right)^{2}} .
$$

Equation (16) shows that $\chi \rightarrow-\infty$ as the parameter approaches the EP $r \rightarrow \pm 1$ which agrees with Eq. (7).

\section{B. Non-Hermitian SSH model}

As a second example we consider the non-Hermitian

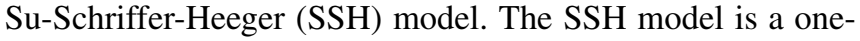
dimensional (1D) fermionic chain with a bond-alternating hopping term, which originally describes the electrons moving in the polyacetylene [44]. The SSH model provides the simplest 1D free fermion model for studying the topological quantum materials. The investigation on the SSH model is widely extended, from the alternating bonds spin- $1 / 2 \mathrm{XXZ}$ chain [45] to 3D topological condensed matter systems, e.g., the Weyl semimetals [46]. Because of its simplicity and nontrivial topological properties, the SSH model has been treated as a parent model for studying non-Hermitian many-body systems [39,47-53]. Since the experimental realization of the non-Hermitian SSH model is easier to set up in a system with a finite number of unit cells with gain and loss, in the following we mainly focus on the finite system with $N$ unit cells, and linking to the thermodynamic limits. The Hamiltonian is

$$
\begin{aligned}
H_{\text {SSH }} & =v \sum_{n=1}^{N}\left(c_{n \uparrow}^{\dagger} c_{n \downarrow}+\text { H.c. }\right)+w \sum_{n=1}^{N}\left(c_{n \downarrow}^{\dagger} c_{n+1 \uparrow}+\text { H.c. }\right) \\
& +i u \sum_{n=1}^{N}\left(c_{n \uparrow}^{\dagger} c_{n \uparrow}-c_{n \downarrow}^{\dagger} c_{n \downarrow}\right),
\end{aligned}
$$

where the parameters $u, v, w \in \mathbb{R}$, and $u \geqslant 0 . c_{n \uparrow}$ and $c_{n \downarrow}$ are the annihilation operators at the $n$th unit cell for the site with gain and loss, respectively. The usual anticommutation relation $\left\{c_{n \sigma}, c_{m \tau}^{\dagger}\right\}=\delta_{n m} \delta_{\sigma \tau}$, and the periodic boundary conditions $c_{N+1 \sigma}=c_{1 \sigma}$, are applied. The phase diagram of the non-Hermitian SSH model in the thermodynamic limits has been studied. The system is in the $\mathcal{P} \mathcal{T}$ symmetric topological phase $w>v+u$, the $\mathcal{P} \mathcal{T}$ broken phase $v-u<w<$ $v+u$, and the $\mathcal{P} \mathcal{T}$ symmetric trivial phase $w<v-u$, respectively [52,53]. By employing Fourier transform $\tilde{c}_{k \sigma}=$ $\frac{1}{\sqrt{N}} \sum_{n=1}^{N} e^{i k n} c_{n \sigma}$, where $k=2 \pi m / N$ and $m=0, \ldots, N-1$. The Hamiltonian becomes

$$
H_{\mathrm{SSH}}=\sum_{k}\left[\begin{array}{cc}
\tilde{c}_{k \uparrow}^{\dagger} & \tilde{c}_{k \downarrow}^{\dagger}
\end{array}\right]\left[\begin{array}{cc}
i u & \xi_{k} \\
\xi_{k}^{*} & -i u
\end{array}\right]\left[\begin{array}{c}
\tilde{c}_{k \uparrow} \\
\tilde{c}_{k \downarrow}
\end{array}\right],
$$

where $\xi_{k}=v+w e^{i k}$. The eigenvalues for the single particle is $\varepsilon_{k}^{ \pm}= \pm \sqrt{\left|\xi_{k}\right|^{2}-u^{2}}$. After constructing the set of operators $\left(\psi_{k}^{\mathrm{R} \pm}\right)^{\dagger}=\left(\varepsilon_{k}^{ \pm}+i u\right) \frac{\xi_{k}}{\left|\xi_{k}\right|^{2}} \tilde{c}_{k \uparrow}^{\dagger}+\tilde{c}_{k \downarrow}^{\dagger}$, and $\psi_{k}^{\mathrm{L} \pm}=\frac{1}{2 \varepsilon_{k}^{ \pm}}\left[\xi_{k}^{*} \tilde{c}_{k \uparrow}+\right.$ $\left.\left(\varepsilon_{k}^{ \pm}-i u\right) \tilde{c}_{k \downarrow}\right]$, the Hamiltonian becomes diagonalized to

$$
H_{\mathrm{SSH}}=\sum_{k}\left[\varepsilon_{k}^{+}\left(\psi_{k}^{\mathrm{R}+}\right)^{\dagger} \psi_{k}^{\mathrm{L}+}+\varepsilon_{k}^{-}\left(\psi_{k}^{\mathrm{R}-}\right)^{\dagger} \psi_{k}^{\mathrm{L}-}\right]
$$

Where $\psi_{k}^{\mathrm{R} \pm}$ and $\psi_{k^{\prime}}^{\mathrm{L} \pm}$ satisfy $\left\langle 0\left|\left(\psi_{k}^{\mathrm{R} \pm}\right)^{\dagger}=0=\psi_{k}^{\mathrm{L} \pm}\right| 0\right\rangle$, $|0\rangle$ is the vacuum state, and $\langle 0 \mid 0\rangle=1$. Moreover, their (anti)commutators with the Hamiltonian are $\left[H_{\mathrm{SSH}},\left(\psi_{k}^{\mathrm{R} \pm}\right)^{\dagger}\right]=\varepsilon_{k}^{ \pm}\left(\psi_{k}^{\mathrm{R} \pm}\right)^{\dagger}, \quad\left[H_{\mathrm{SSH}}, \psi_{k}^{\mathrm{L} \pm}\right]=\varepsilon_{k}^{ \pm} \psi_{k}^{\mathrm{L} \pm}$, and $\left\{\psi_{k}^{\mathrm{L} \tau},\left(\psi_{k^{\prime}}^{\mathrm{R} \tau^{\prime}}\right)^{\dagger}\right\}=\delta_{k k^{\prime}} \delta^{\tau \tau^{\prime}}$.

The time-dependent metric tensor can be chosen to be

$$
G(t)=\sum_{p, q=0}^{N} \sum_{k, k^{\prime}}\left(\prod_{a=1}^{p} e^{-2 t \operatorname{Im} \varepsilon_{k_{a}}^{+}}\right)\left(\prod_{b=1}^{q} e^{-2 t \operatorname{Im} \varepsilon_{k_{b}^{\prime}}^{\prime}}\right)\left(\prod_{a=1}^{p} \psi_{k_{a}}^{\mathrm{L}+}\right)^{\dagger}\left(\prod_{b=1}^{q} \psi_{k_{b}^{\prime}}^{\mathrm{L}-}\right)^{\dagger}|0\rangle\langle 0|\left(\prod_{b=1}^{q} \psi_{k_{b}^{\prime}}^{\mathrm{L}-}\right)\left(\prod_{a=1}^{p} \psi_{k_{a}}^{\mathrm{L}+}\right),
$$

where

$$
\sum_{k, k^{\prime}} \equiv \sum_{k_{1}=1}^{N} \sum_{k_{2}=k_{1}+1}^{N} \cdots \sum_{k_{p}=k_{p-1}+1}^{N} \sum_{k_{1}^{\prime}=1}^{N} \sum_{k_{2}^{\prime}=k_{1}^{\prime}+1}^{N} \cdots \sum_{k_{q}^{\prime}=k_{q-1}^{\prime}+1}^{N}
$$

From Eq. (19) we define that the half-filled right eigenstate $\left.\left|\Psi_{0}\right\rangle\right\rangle$ such that $\left.\left(\psi_{k}^{\mathrm{R}-}\right)^{\dagger}\left|\Psi_{0}\right\rangle\right\rangle=0$ for all $k$ is the "ground state." In other words, the ground state is the state full of particles in the $\varepsilon_{k}^{-}$band, even $\varepsilon_{k}^{-}$can be complex in the $\mathcal{P} \mathcal{T}$ broken phase. The time-dependent right ground state is

$$
\left.\left|\Psi_{0}(t)\right\rangle\right\rangle=\left[\prod_{k} e^{-i t \varepsilon_{k}^{-}}\left(\psi_{k}^{\mathrm{R}-}\right)^{\dagger}\right]|0\rangle,
$$

and the corresponding dual vector of the right ground state $\left\langle\left\langle\Psi_{0}(t)\right|=\left\langle\Psi_{0}(t)\right| G(t)\right.$ is the left ground state,

$$
\left\langle\left\langle\Psi_{0}(t)\right|=\langle 0|\left(\prod_{k} e^{i t \varepsilon_{k}^{-}} \psi_{k}^{\mathrm{L}-}\right) .\right.
$$

The fidelity susceptibility density $\chi_{0}$ for the two ground states with parameters $(u, v, w)$ and $(u, v, w+\epsilon)$ is

$$
\chi_{0}=\frac{1}{N} \sum_{k} \frac{v^{2} \sin ^{2} k-u^{2}}{4\left(v^{2}+w^{2}+2 v w \cos k-u^{2}\right)^{2}},
$$

where $\epsilon$ is taken to be infinitesimally small. It is clear that $\chi_{0}$ is a time-independent quantity.

In the case $u=0$, the Hermitian limit, the ground state fidelity susceptibility density Eq. (23) for $v=1$ is plotted in 

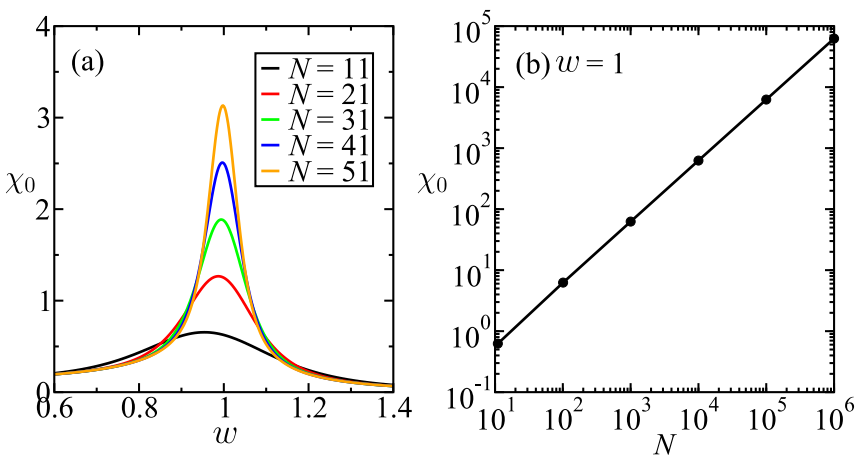

FIG. 1. Ground state fidelity susceptibility density $\chi_{0}$ for the Hermitian $(u=0)$ SSH model. $v=1$ is set as the unit. (a) $\chi_{0}$ as a function of $w$. (b) The size dependence at the quantum critical point $w=1$ is plotted, and a power-law divergence is found, $\chi_{0}=$ $5(N-1) / 80$.

Fig. 1. For $N$ is even, there is a $k=\pi$ such that Eq. (23) diverges at the critical point $(u, v, w)=(0,1,1)$. Therefore we focus on the odd $N$ for looking at the size dependencies, where $\chi_{0}$ is always finite. It is known that $\chi_{0}$ goes to $+\infty$ at the quantum critical point $w=v$ in the thermodynamic limit $N \rightarrow \infty$. The scaling properties of the fidelity susceptibility have been studied for the quantum phase transitions [54]. From Eq. (23) we obtain a power-law divergence $\chi_{0}=$ $5(N-1) / 80$ at $v=w=1$, as shown in Fig. 1(b).

For the non-Hermitian $(u \neq 0)$ case, as $|w-v| \leqslant|u|$, there is a $k=k_{\mathrm{EP}}$ such that the denominator vanishes. Since the denominator is always positive and the numerator is always negative, the ground state fidelity susceptibility density $\chi_{0} \rightarrow-\infty$ characterizes an EP if one of the $k$ points closes to the $k_{\mathrm{EP}}$. Therefore in the thermodynamics limits, i.e., the values of $k$ are continuous in $[0,2 \pi)$, the $\mathcal{P} \mathcal{T}$ broken phase $v-u \leqslant w \leqslant v+u$ becomes the exceptional line. In other words, the exceptional line is an emergent phenomena in the thermodynamics limits, while in the finite system the exceptional line breaks down into separate EPs. For example, as shown in Fig. 2(a), the exceptional line in the finite size system $N=101$ breaks down into two EPs and four EPs for $u=0.04$ and $u=0.1$, respectively. As the parameter
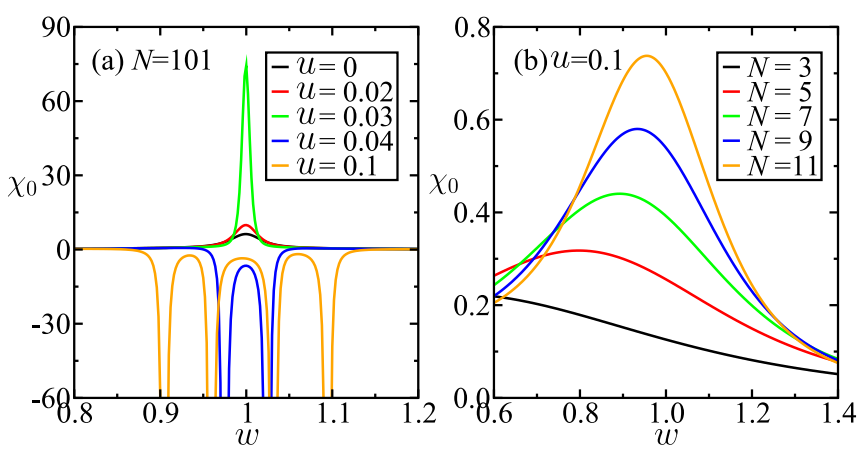

FIG. 2. Ground state fidelity susceptibility density $\chi_{0}$ for the non-Hermitian SSH model. $v=1$ is set as the unit. (a) For $N=101$, the signal of the Hermitian quantum phase transition is enhanced by adding a small non-Hermitian term. The EP is not present in the finite $N=101$ until $u \gtrsim 0.04$. (b) For $u=0.1$, small finite size $N$ would lead the absence of the EP. approaches these EPs, the behavior of $\chi_{0}$ agrees with the hypothesis Eq. (7).

Interestingly, since there is only a limited number of $k$ points in the finite size system, we find no EP at all in the whole parameter space if the non-Hermitian driving parameter $u$ is small enough or the size $N$ is odd and small. As shown in Fig. 2(a) for larger size $N=101$ and smaller sizes in Fig. 2(b), the ground state fidelity susceptibility density $\chi_{0}$ is always positive and finite for $u \lesssim 0.03$ with $N=101$ system and $u=0.1$ for smaller systems, showing no EP in the whole parameter space. The absence of EP means that these finite size systems are $\mathcal{P} \mathcal{T}$ preserving in the whole $(v, w)$ parameter region, i.e., the $\mathcal{P} \mathcal{T}$ breaking phase vanishes in the odd $N$ systems. Although the terminology "phases of matter" implies the case is in the thermodynamic limits, the system with size $N=101$ is usually large enough for distinguishing different phases in many numerical studies about phase transitions. Our result shows that $\mathcal{P} \mathcal{T}$ breaking region does not exist for finite size systems provided $u$ is small enough, which further extends the discussions in the previous findings [52,53]. For example, $u=0.1$, the small size systems in Fig. 2(b) have no EP. However, once the size is large enough, at least two EPs are present at the phase boundary, as shown in Fig. 2(a). For $u \leqslant 0.03$, the size should be much larger than $N=101$ for opening the $\mathcal{P} \mathcal{T}$ breaking region. Because the sensitivity of the non-Hermitian system could be enhanced near the EP $[29,38]$, the small $u$, which is not strong enough to open the $\mathcal{P} \mathcal{T}$ breaking region, consequently, enhances the signal of the phase transitions between the topological phase and the trivial phase. As shown in Fig. 2(a), $\chi_{0}$ with small $u$ becomes much larger than the case $u=0$. The amplified susceptibility means that by a small non-Hermitian perturbation, the finite size system becomes more sensitive in responding to the quantum phase transition. In other words, we present an example that a small non-Hermitian perturbation is helpful to detect quantum phase transitions. For those with particular difficulty, e.g., higher-order quantum phase transitions [42] and the Kosterlitz-Thouless transition [55-57], further investigations on the generalized fidelity susceptibility are interesting and remain as open questions.

\section{CONCLUSION}

We have properly generalized the fidelity Eq. (3) for nonHermitian quantum systems by including the metric of the Hilbert space. We mention that, unlike the phase transition, the EP is not a thermodynamic but the geometric property of the Hilbert space. In the perspective of the fidelity susceptibility, both the EP and the quantum critical point enhance the sensitivity of a non-Hermitian system, however the EP is a geometric effect and divergence direction is clearly distinguished from the quantum critical point. We propose that Eq. (7) is one of the basic properties of EPs. Since the previous study of the fidelity shows the failure of detecting some quantum phase transitions [42], this hypothesis for the EPs might also break down in some non-Hermitian systems. However, we have not found any counterexample against Eq. (7).

Moreover, since the computation of the generalized fidelity is reduced to the biorthogonal formalism Eq. (5) if the parameter is not the EP [41], numerical computations for 
more complicated systems require only one of the biorthogonal eigenpair of the non-Hermitian Hamiltonian, instead of solving the metric equation of motion Eq. (1) or full diagonalization.

\section{ACKNOWLEDGMENTS}

Y.-C.T. is grateful to Min-Fong Yang and Po-Yao Chang for many useful discussions. Y.-C.T. acknowledges sup- port by the Ministry of Science and Technology of Taiwan (MOST) under Grants No. MOST 108-2811-M-005-522, No. 108-2112-M-029-002, and No.109-2636-M-007-003. W.-M.H. and C.-Y.J. are supported by the MOST through 107-2112-M-005-008-MY3 and 109-2811-M-005-509, respectively. G.-Y.C. and C.-Y.J. are supported partially by the MOST, through Grant No. MOST 109-2112-M-005-002.

Y.-C.T. and C.-Y.J. contributed equally to this work.
[1] S.-J. Gu, Fidelity approach to quantum phase transitions, Int. J. Mod. Phys. B 24, 4371 (2010).

[2] R. Jozsa, Fidelity for mixed quantum states, J. Mod. Opt. 41, 2315 (1994).

[3] C. M. Bender and S. Boettcher, Real Spectra in Non-Hermitian Hamiltonians having $\mathcal{P} \mathcal{T}$ symmetry, Phys. Rev. Lett. 80, 5243 (1998).

[4] B. Peng, Ş. K. Özdemir, F. Lei, F. Monifi, M. Gianfreda, G. L. Long, S. Fan, F. Nori, C. M. Bender, and L. Yang, Parity-timesymmetric whispering-gallery microcavities, Nat. Phys. 10, 394 (2014).

[5] H. Jing, S. K. Özdemir, X.-Y. Lü, J. Zhang, L. Yang, and F. Nori, $\mathcal{P} \mathcal{T}$-Symmetric Phonon Laser, Phys. Rev. Lett. 113, 053604 (2014).

[6] H. Jing, Ş. K. Özdemir, Z. Geng, J. Zhang, X.-Y. Lü, B. Peng, L. Yang, and F. Nori, Optomechanically-induced transparency in parity-time-symmetric microresonators, Sci. Rep. 5, 9663 (2015).

[7] J. Zhang, B. Peng, Ş. K. Özdemir, Y.-X. Liu, H. Jing, X.-Y. Lü, Y.-L. Liu, L. Yang, and F. Nori, Giant nonlinearity via breaking parity-time symmetry: A route to low-threshold phonon diodes, Phys. Rev. B 92, 115407 (2015).

[8] Y.-L. Liu, R. Wu, J. Zhang, Ş. K. Özdemir, L. Yang, F. Nori, and Y.-X. Liu, Controllable optical response by modifying the gain and loss of a mechanical resonator and cavity mode in an optomechanical system, Phys. Rev. A 95, 013843 (2017).

[9] F. Quijandría, U. Naether, Ş. K. Özdemir, F. Nori, and D. Zueco, $\mathcal{P} \mathcal{T}$-symmetric circuit QED, Phys. Rev. A 97, 053846 (2018).

[10] M. Ezawa, Electric circuits for non-Hermitian Chern insulators, Phys. Rev. B 100, 081401(R) (2019).

[11] K. Y. Bliokh, D. Leykam, M. Lein, and F. Nori, Topological non-Hermitian origin of surface Maxwell waves, Nat. Commun. 10, 580 (2019).

[12] Y. Ashida, S. Furukawa, and M. Ueda, Parity-time-symmetric quantum critical phenomena, Nat. Commun. 8, 15791 (2017).

[13] K. Y. Bliokh and F. Nori, Klein-Gordon Representation of Acoustic Waves and Topological Origin of Surface Acoustic Modes, Phys. Rev. Lett. 123, 054301 (2019).

[14] K. Jones-Smith and H. Mathur, Relativistic non-Hermitian quantum mechanics, Phys. Rev. D 89, 125014 (2014).

[15] H. Zhao, X. Qiao, T. Wu, B. Midya, S. Longhi, and L. Feng, Non-Hermitian topological light steering, Science 365, 1163 (2019).

[16] R. Hanai, A. Edelman, Y. Ohashi, and P. B. Littlewood, NonHermitian Phase Transition from a Polariton Bose-Einstein Condensate to a Photon Laser, Phys. Rev. Lett. 122, 185301 (2019).
[17] R. Hanai and P. B. Littlewood, Critical fluctuations at a many-body exceptional point, Phys. Rev. Res. 2, 033018 (2020).

[18] Ş. K. Özdemir, S. Rotter, F. Nori, and L. Yang, Parity-time symmetry and exceptional points in photonics, Nat. Mater. 18, 783 (2019).

[19] Y.-C. Lee, M.-H. Hsieh, S. T. Flammia, and R.-K. Lee, Local $\mathcal{P} \mathcal{T}$ Symmetry Violates the No-Signaling Principle, Phys. Rev. Lett. 112, 130404 (2014).

[20] S.-L. Chen, G.-Y. Chen, and Y.-N. Chen, Increase of entanglement by local $\mathcal{P} \mathcal{T}$-symmetric operations, Phys. Rev. A 90 , 054301 (2014).

[21] A. Mostafazadeh, Time-dependent pseudo-Hermitian Hamiltonians defining a unitary quantum system and uniqueness of the metric operator, Phys. Lett. B 650, 208 (2007).

[22] B. Gardas, S. Deffner, and A. Saxena, Non-Hermitian quantum thermodynamics, Sci. Rep. 6, 23408 (2016).

[23] C.-Y. Ju, A. Miranowicz, G.-Y. Chen, and F. Nori, NonHermitian Hamiltonians and no-go theorems in quantum information, Phys. Rev. A 100, 062118 (2019).

[24] D. C. Brody, Biorthogonal quantum mechanics, J. Phys. A: Math. Theor. 47, 035305 (2013).

[25] T. Kato, Perturbation Theory for Linear Operators (Springer, Berlin, 1966), Vol. 132.

[26] W. D. Heiss, The physics of exceptional points, J. Phys. A 45, 444016 (2012).

[27] M.-A. Miri and A. Alù, Exceptional points in optics and photonics, Science 363, eaar7709 (2019).

[28] E. J. Bergholtz, J. C. Budich, and F. K. Kunst, Exceptional topology of non-Hermitian systems, arXiv:1912.10048.

[29] H. Hodaei, A. U. Hassan, S. Wittek, H. Garcia-Gracia, R El-Ganainy, D. N. Christodoulides, and M. Khajavikhan, Enhanced sensitivity at higher-order exceptional points, Nature (London) 548, 187 (2017).

[30] J.-H. Park, A. Ndao, W. Cai, L.-Y. Hsu, A. Kodigala, T. Lepetit, Y.-H. Lo, and B. Kanté, Observation of plasmonic exceptional points, arXiv:1904.01073.

[31] J. Doppler, A. A. Mailybaev, J. Böhm, U. Kuhl, A. Girschik, F. Libisch, T. J. Milburn, P. Rabl, N. Moiseyev, and S. Rotter, Dynamically encircling an exceptional point for asymmetric mode switching, Nature (London) 537, 76 (2016).

[32] H. Xu, D. Mason, L. Jiang, and J. G. E. Harris, Topological energy transfer in an optomechanical system with exceptional points, Nature (London) 537, 80 (2016).

[33] D. Zhang, X.-Q. Luo, Y.-P. Wang, T.-F. Li, and J. Q. You, Observation of the exceptional point in cavity magnon-polaritons, Nat. Commun. 8, 1 (2017). 
[34] M. Harder, Y. Yang, B. M. Yao, C. H. Yu, J. W. Rao, Y. S. Gui, R. L. Stamps, and C.-M. Hu, Level Attraction Due to Dissipative Magnon-Photon Coupling, Phys. Rev. Lett. 121, 137203 (2018).

[35] J. Xu, Y.-X. Du, W. Huang, and D.-W. Zhang, Detecting topological exceptional points in a parity-time symmetric system with cold atoms, Opt. Express 25, 15786 (2017).

[36] T. Yoshida, R. Peters, N. Kawakami, and Y. Hatsugai, Symmetry-protected exceptional rings in two-dimensional correlated systems with chiral symmetry, Phys. Rev. B 99, 121101(R) (2019).

[37] K. Kimura, T. Yoshida, and N. Kawakami, Chiral-symmetry protected exceptional torus in correlated nodal-line semimetals, Phys. Rev. B 100, 115124 (2019).

[38] W. Chen, Ş. K. Özdemir, G. Zhao, J. Wiersig, and L. Yang, Exceptional points enhance sensing in an optical microcavity, Nature (London) 548, 192 (2017).

[39] H. Jiang, C. Yang, and S. Chen, Topological invariants and phase diagrams for one-dimensional two-band non-Hermitian systems without chiral symmetry, Phys. Rev. A 98, 052116 (2018).

[40] N. Matsumoto, K. Kawabata, Y. Ashida, S. Furukawa, and M. Ueda, Continuous Phase Transition without Gap Closing in Non-Hermitian Quantum Many-Body Systems, Phys. Rev. Lett. 125, 260601 (2020).

[41] The definition of the generalized fidelity Eq. (3) still holds at the EP, however one should solve the equation of motion Eq. (1) for finding a valid metric. The biorthogonal fidelity in Eq. (5) is only convenient for calculation outside the EP. At the EP it loses the geometric meaning of the Hilbert space. Here we investigate the behavior of the biorthogonal fidelity susceptibility $\chi$ as the parameter approaches the EP.

[42] Note that some higher order quantum phase transitions fail to be detected by the fidelity susceptibility, see Y.-C. Tzeng, H.-H. Hung, Y.-C. Chen, and M.-F. Yang, Fidelity approach to Gaussian transitions, Phys. Rev. A 77, 062321 (2008).

[43] C. M. Bender, D. C. Brody, and H. F. Jones, Complex Extension of Quantum Mechanics, Phys. Rev. Lett. 89, 270401 (2002).

[44] W. P. Su, J. R. Schrieffer, and A. J. Heeger, Solitons in Polyacetylene, Phys. Rev. Lett. 42, 1698 (1979).
[45] Y.-C. Tzeng, L. Dai, M. Chung, L. Amico, and L.-C. Kwek, Entanglement convertibility by sweeping through the quantum phases of the alternating bonds XXZ chain, Sci. Rep. 6, 26453 (2016).

[46] Y.-C. Tzeng and M.-F. Yang, Fate of Fermi-arc states in gapped Weyl semimetals under long-range interactions, Phys. Rev. B 102, 035148 (2020).

[47] W. Song, W. Sun, C. Chen, Q. Song, S. Xiao, S. Zhu, and T. Li, Breakup and Recovery of Topological Zero Modes in Finite Non-Hermitian Optical Lattices, Phys. Rev. Lett. 123, 165701 (2019).

[48] M. Parto, S. Wittek, H. Hodaei, G. Harari, M. A. Bandres, J. Ren, M. C. Rechtsman, M. Segev, D. N. Christodoulides, and M. Khajavikhan, Edge-Mode Lasing in 1D Topological Active Arrays, Phys. Rev. Lett. 120, 113901 (2018).

[49] S. Yao and Z. Wang, Edge States and Topological Invariants of Non-Hermitian Systems, Phys. Rev. Lett. 121, 086803 (2018).

[50] M. Pan, H. Zhao, P. Miao, S. Longhi, and L. Feng, Photonic zero mode in a non-Hermitian photonic lattice, Nat. Commun. 9, 1 (2018).

[51] L. Herviou, N. Regnault, and J. H. Bardarson, Entanglement spectrum and symmetries in non-Hermitian fermionic noninteracting models, SciPost Phys. 7, 69 (2019).

[52] S. Lieu, Topological phases in the non-Hermitian Su-SchriefferHeeger model, Phys. Rev. B 97, 045106 (2018).

[53] P.-Y. Chang, J.-S. You, X. Wen, and S. Ryu, Entanglement spectrum and entropy in topological non-Hermitian systems and nonunitary conformal field theory, Phys. Rev. Res. 2, 033069 (2020).

[54] Y.-C. Tzeng and M.-F. Yang, Scaling properties of fidelity in the spin-1 anisotropic model, Phys. Rev. A 77, 012311 (2008).

[55] J. M. Kosterlitz and D. J. Thouless, Ordering, metastability and phase transitions in two-dimensional systems, J. Phys. C 6, 1181 (1973).

[56] M.-F. Yang, Ground-state fidelity in one-dimensional gapless models, Phys. Rev. B 76, 180403(R) (2007).

[57] G. Sun, A. K. Kolezhuk, and T. Vekua, Fidelity at BerezinskiiKosterlitz-Thouless quantum phase transitions, Phys. Rev. B 91, 014418 (2015). 